\title{
Nonlinear free vibration of single walled Carbone NanoTubes conveying fluid
}

\author{
A. Azrar ${ }^{1}$, L. Azrar ${ }^{1,2}$, and A. A. Aljinaidi ${ }^{2}$ \\ ${ }^{1}$ Mathematical Modeling and Control, Faculty of Sciences and Techniques of Tangier \\ University Abdelmalek Essaadi, BP 416, Tangier, Morocco. \\ ${ }^{2}$ Departement of Mechanical Engineering, Faculty of Engineering, Kind Abdulaziz \\ University, Jeddah, Saudi Arabia
}

\begin{abstract}
Nonlinear free vibration of single-walled carbon nanotubes (CNTs) conveying fluid are modeled and numerically simulated based on von Kármán geometric nonlinearity and Eringen's nonlocal elasticity theory. The CNTs are modelled as nanobeams where the effects of transverse shear deformation and rotary inertia are considered within the framework of Timoshenko beam theory. The governing equations and boundary conditions are derived using the Hamilton's principle and the nonlinear equation of motion is solved by the Galerkin's method. The small scale parameter and the fluid-tube interaction effects on the dynamic behaviours of the CNT-fluid system as well as the instabilities induced by the fluid-velocity can be investigated. The critical fluidvelocity and frequency-amplitude relationships as well as the flutter and divergence instability types and the associated time responses are obtained based on the presented methodological approach.
\end{abstract}

\section{Introduction}

Vibration analysis of CNTs conveying fluid has been one of the attractive subjects in structural dynamics. This is because CNTs promise many applications in nanobiological devices and nanomechanical systems. The theory of nonlocal elasticity, Eringen [1], allows accounting for the small scale effect that is very significant when dealing with micro and nanostructures Yang et al [2], developed the nonlinear free vibration of single-walled carbon nanotubes using nonlocal Timoshenko beam theory, Wang et al [3], discussed the nonlinear vibration of embedded single-walled carbon nanotube with geometrical imperfection under harmonic load based on nonlocal Timoshenko beam theory. Many research studies have reported the influence of fluid flow on the mechanical properties of CNT. Lee and Chang [4], studied the vibration analysis of a viscous-fluid-conveying single-walled carbon nanotube embedded in an elastic medium. Length scale effect analysis on vibration behavior of single walled Carbon NanoTubes with various boundary condition types have been elaborated by Azrar et al $[5,6]$.

In this paper, the small scale parameter and the internal fluid interaction effects on the dynamic behaviors of the single walled CNT-fluid system as well as the instabilities induced by the fluid-velocity are investigated. The geometric nonlinearity in the von Kármán type is included and contributions of fluid velocity to the kinetic energy as well as to the body forces are accounted for.

\section{Mathematical formulation}

Let us consider a slender single walled carbon nanotube of length $\mathrm{L}$, diameter $\mathrm{d}$ and thickness $\mathrm{h}$. The nonlocal elasticity theory combined with the Timoshenko beam model and nonlinear effect are adopted. The dynamic governing equations are formulated.

\subsection{Governing dynamic equations}

Based on the nonlocal elasticity theory, developed by Eringen [1], the constitutive equation for a linear homogenous nonlocal elastic body is given by the following integral equation:

$$
\begin{aligned}
& \sigma_{i j}(x)=\int_{V} \alpha\left(\left|x-x^{\prime}\right|, \tau\right) C_{i j k l} \varepsilon_{k l}\left(x^{\prime}\right) d V\left(x^{\prime}\right), \\
& \varepsilon_{k l}=\left(u_{k, l}+u_{l, k}\right) / 2
\end{aligned}
$$

in which $\alpha\left(\left|x-x^{\prime}\right|, \tau\right)$ is the nonlocal kernel function, which incorporates the nonlocal effect at the reference point $\mathrm{x}$ produced by local strain point at the source $\mathrm{x}$ ' into the constitutive equations; $\tau=e_{0} a / L$ where a and $e_{0}$ are a constant appropriate to each material, an internal 
characteristic length and $\mathrm{L}$ is the length. $C_{i j k l}$ is the elastic modulus tensor, $\sigma_{i j}$ and $\varepsilon_{i j}$ are the stress and the strain tensors. The nonlocal kernel function $\alpha$ depends on the internal and external characteristics lengths. Various approximate models of nonlocal elasticity can be obtained by specifying the kernel function $\alpha$. Based on the von Karman model the nonlinear strain-displacement relation is given by:

$\varepsilon_{x x}=\varepsilon_{x x}^{0}+z \frac{\partial \psi}{\partial x}, \varepsilon_{x x}^{0}=\frac{\partial u}{\partial x}+\frac{1}{2}\left(\frac{\partial w}{\partial x}\right)^{2}, \gamma_{x z}=\psi-\frac{\partial w}{\partial x}$,

where $\psi$ denotes the rotation of the cross section, $u$ and $w$ are the axial and transverse displacements in the middle plane $(\mathrm{z}=0)$ and $t$ is time. Using these equations, the nonlocal resultant axial force $N_{x}$, moment $M_{x}$ and shear force $Q_{\mathrm{x}}$ are given by the following differential equations [2].

$$
\begin{aligned}
& N_{x}=\left(e_{0} a\right)^{2} \frac{\partial^{2} N_{x}}{\partial x^{2}}+E A\left[\frac{\partial u}{\partial x}+\frac{1}{2}\left(\frac{\partial w}{\partial x}\right)^{2}\right], \\
& M_{x}=\left(e_{0} a\right)^{2} \frac{\partial^{2} M_{x}}{\partial x^{2}}+E I \frac{\partial \psi}{\partial x}, \\
& Q_{x}=\left(e_{0} a\right)^{2} \frac{\partial^{2} Q_{x}}{\partial x^{2}}+k s A G\left(\psi-\frac{\partial w}{\partial x}\right),
\end{aligned}
$$

in which

$$
N=\int_{A} \sigma_{x x} d A, \quad M=\int_{A} z \sigma_{x x} d A, Q=k s \int_{A} \sigma_{x z} d A
$$

where $k s$ is the factor of shear depending on the shape of the cross section $A$ and its value is $k s=2(1+v) /(4+3 v)$.

$I, E$ and $G$ are the second moment of inertia of the nanotube, Young and shear modulus respectively.

When formulating the equations of motion for elastic beam. The fluid interaction force is given by:

$$
\Pi=m_{f}\left(\frac{\partial}{\partial t}+v \frac{\partial}{\partial x}\right)^{2} w
$$

The variation of the strain energy $\delta \Pi$ is given by

$$
\begin{aligned}
& \delta \Pi=\frac{1}{2} \iint_{0}^{L} \int_{A}\left(\sigma_{x x} \delta \varepsilon_{x x}+\sigma_{x z} \delta \gamma_{x z}\right) d x d A+\int_{0}^{L}\left(K_{w}-q\right) \delta w d x \\
& +\int_{0}^{L} m_{f} v^{2} \frac{\partial^{2} w}{\partial x^{2}}\left(\delta w-\frac{\partial w}{\partial x} \delta u\right) d x+\int_{0}^{L} m_{f} v\left(\delta u-\frac{\partial w}{\partial x} \delta w\right) d x(7)
\end{aligned}
$$

The variation of the kinetic energy $\delta K$ is given by:

$$
\begin{aligned}
& \delta K=\frac{1}{2} \int_{0}^{L} \int_{A} \rho_{c}[(\dot{u}+z \dot{\psi})(\delta \dot{u}+z \delta \dot{\psi})+\dot{w} \delta \dot{w}] d A d x \\
& +\int_{0}^{L} \int_{A f} \rho_{f}\left[(\dot{u}+v) \delta(\dot{u}+v)+\left(v \frac{\partial w}{\partial x}+\dot{w}\right) \delta\left(v \frac{\partial w}{\partial x}+\dot{w}\right)\right] d A d x(8)
\end{aligned}
$$

Using the Hamilton's principle

$$
\int_{0}^{t}\left(\delta K+\delta V_{p}\right) d t=0
$$

Substituting Eqs.(7) and (8) into Eq.(9), integrating by parts and setting the coefficients of $\delta u, \delta w$ and $\delta \psi$ to zero lead to the following partial differential system:

$$
\begin{aligned}
& \frac{\partial N_{x}}{\partial x}=\left(m_{c}+m_{f}\right) \frac{\partial^{2} u}{\partial t^{2}}-m_{f} v \frac{\partial w}{\partial x}\left(\frac{\partial^{2} w}{\partial t \partial x}+v \frac{\partial^{2} w}{\partial x^{2}}\right), \\
& \frac{\partial Q_{x}}{\partial x}+\frac{\partial}{\partial x}\left(N_{x} \frac{\partial w}{\partial x}\right)-k_{w} w+q(x, t)=\left(m_{c}+m_{f}\right) \frac{\partial^{2} w(x, t)}{\partial t^{2}} \\
& +m_{f} v^{2} \frac{\partial^{2} w}{\partial x^{2}}+m_{f} v\left(2 \frac{\partial^{2} w}{\partial x \partial t}-\frac{\partial u}{\partial t} \frac{\partial^{2} w}{\partial x^{2}}-\frac{\partial w}{\partial x} \frac{\partial w}{\partial t} \frac{\partial^{2} w}{\partial x^{2}}-\frac{\partial w}{\partial x} \frac{\partial^{2} u}{\partial x \partial t}\right),(10 b) \\
& \frac{\partial M}{\partial x}-Q(x, t)=\left(J_{c}+J_{f}\right) \frac{\partial^{2} \psi}{\partial t^{2}},
\end{aligned}
$$

where $k_{w}, q(x, t), v$ are the Winkler constant of the surrounding elastic medium described as a Winkler-like elastic foundation, the transverse excitation force per unit length and $\mathrm{v}$ is the uniform mean flow velocity of conveying fluid. For a constant cross section, the mass inertia $m_{c}$ and $m_{f}$ are the mass per unit length for CNT and fluid respectively, $J_{c}$ and $J_{f}$ are the mass moment and inertia for CNT and fluid respectively.

Based on the equations (4) and (10), explicit expressions of the nonlocal resultant force $N_{x}$, bending moment $M_{x}$ and shear force $Q_{\mathrm{x}}$ obtained with respect to displacements by:

$$
\begin{aligned}
N_{x}= & E A\left[\frac{\partial u}{\partial x}+\frac{1}{2}\left(\frac{\partial^{2} w}{\partial x^{2}}\right)^{2}\right]+\left(e_{0} a\right)^{2}\left[\left(m_{c}+m_{f}\right) \frac{\partial^{3} u}{\partial x \partial t^{2}}\right. \\
& \left.-m_{f} v \frac{\partial}{\partial x}\left[\frac{\partial w}{\partial x}\left(\frac{\partial^{2} w}{\partial t \partial x}+v \frac{\partial^{2} w}{\partial x^{2}}\right)\right]\right], \\
M_{x}= & E I \frac{\partial \psi}{\partial x}+\left(e_{0} a\right)^{2}\left[\left(J_{c}+J_{f}\right) \frac{\partial^{3} \psi(x, t)}{\partial x \partial t^{2}}+\left(m_{c}+m_{f}\right) \frac{\partial^{2} w}{\partial t^{2}}\right. \\
& -\frac{\partial}{\partial x}\left(N_{x} \frac{\partial w}{\partial x}\right)+K_{w} w-q+m_{f} V^{2} \frac{\partial^{2} w}{\partial x^{2}} \\
& \left.+m_{f} v\left(2 \frac{\partial^{2} w}{\partial x \partial t}-\frac{\partial u}{\partial t} \frac{\partial^{2} w}{\partial x^{2}}-\frac{\partial w}{\partial x} \frac{\partial w}{\partial t} \frac{\partial^{2} w}{\partial x^{2}}-\frac{\partial w}{\partial x} \frac{\partial^{2} u}{\partial x \partial t}\right)\right] \\
Q_{x}= & K s A G\left(\psi-\frac{\partial w}{\partial x}\right)+\left(e_{0} a\right)^{2}\left[\left(m_{c}+m_{f}\right) \frac{\partial^{3} w(x, t)}{\partial x \partial t^{2}}+m_{f} V^{2} \frac{\partial^{3} w}{\partial x^{3}}\right. \\
& \left.-\frac{\partial^{2}}{\partial x^{2}}\left(N_{x} \frac{\partial w}{\partial x}\right)+K_{w} \frac{\partial w}{\partial x}-\frac{\partial q}{\partial x}\right]+m_{f} v \frac{\partial}{\partial x}\left(2 \frac{\partial^{2} w}{\partial x \partial t}-\frac{\partial u}{\partial t} \frac{\partial^{2} w}{\partial x^{2}}\right. \\
& \left.-\frac{\partial w}{\partial x} \frac{\partial w}{\partial t} \frac{\partial^{2} w}{\partial x^{2}}-\frac{\partial w}{\partial x} \frac{\partial^{2} u}{\partial x \partial t}\right),
\end{aligned}
$$

Further developments lead to the nonlinear partial differential system governing the dynamic behavior of undamped CNTs conveying fluid. For dimensionless equations, the following variables are used.

$$
\begin{aligned}
& y=\frac{x}{L} ; W=\frac{w}{L} ; \tau=\frac{L}{d} ; \mu=\frac{e_{0} a}{L} ; \xi=\frac{E A L^{2}}{E I} ; q(x, t)=0 ; \chi=\frac{4+3 v}{8 \tau^{2}} ; \\
& m=\frac{m_{f}}{\left(m_{c}+m_{f}\right)} ; V=\sqrt{\frac{m_{f}}{E I}} \nu L ; \beta=\sqrt{\frac{\left(m_{c}+m_{f}\right) L^{4}}{E I}} ; \delta=1 / 4 \tau^{2}\left(1-\frac{m}{2}\right)(15)
\end{aligned}
$$

The resulting coupled nonlinear partial differential equations are then obtained. 


$$
\left\{\begin{array}{l}
\beta^{2} \frac{\partial^{2}}{\partial t^{2}}\left(1-\mu^{2} \frac{\partial^{2} U}{\partial y^{2}}\right)-\xi \frac{\partial^{2} U}{\partial y^{2}}=\xi\left(\frac{\partial^{2} W}{\partial y^{2}} \frac{\partial^{3} W}{\partial y^{3}}\right)+\beta V \sqrt{m}\left[\frac{\partial W}{\partial y} \frac{\partial^{2} W}{\partial t \partial y}\right. \\
\left.-\mu^{2}\left(\frac{\partial^{3} W}{\partial y^{3}} \frac{\partial^{2} W}{\partial t \partial y}+\frac{\partial W}{\partial y} \frac{\partial^{4} W}{\partial t \partial y^{3}}\right)\right]+V^{2}\left[\frac{\partial W}{\partial y} \frac{\partial^{2} W}{\partial y^{2}}+\left(\frac{\partial^{3} W}{\partial y^{3}} \frac{\partial^{2} W}{\partial y^{2}}+\frac{\partial W}{\partial y} \frac{\partial^{4} W}{\partial y^{4}}\right)\right],(16 a) \\
\frac{\partial}{\partial y}\left(\Psi-\frac{\partial W}{\partial y}\right)+\chi \xi\left(\frac{\partial^{2} U}{\partial y^{2}} \frac{\partial W}{\partial y}+\frac{3}{2}\left(\frac{\partial W}{\partial y}\right)^{2} \frac{\partial^{2} W}{\partial y^{2}}+\frac{\partial U}{\partial y} \frac{\partial^{2} W}{\partial y^{2}}\right) \\
-\mu^{2} \chi \xi\left[\frac{\partial^{4} U}{\partial y^{4}} \frac{\partial W}{\partial y}+3 \frac{\partial^{3} U}{\partial y^{3}} \frac{\partial^{2} W}{\partial y^{2}}+3 \frac{\partial^{2} U}{\partial y^{2}} \frac{\partial^{3} W}{\partial y^{3}}+\frac{\partial U}{\partial y} \frac{\partial^{4} W}{\partial y^{4}}+\left(\frac{\partial^{2} W}{\partial y^{2}}\right)^{3}\right. \\
\left.\left.+9 \frac{\partial W}{\partial y} \frac{\partial^{2} W}{\partial y^{2}} \frac{\partial^{3} W}{\partial y^{3}}+\frac{3}{2}\left(\frac{\partial W}{\partial y}\right)^{2} \frac{\partial^{4} W}{\partial y^{4}}\right)\right]+\chi\left(\frac{\tilde{q}-\mu^{2}}{\partial y^{2}}\right)+K_{w} \chi\left(W-\mu^{2} \frac{\partial^{2} W}{\partial y^{2}}\right) \\
+\chi \beta^{2} \frac{\partial^{2}}{\partial t^{2}}\left(W-\mu^{2} \frac{\partial^{2} W}{\partial y^{2}}\right)-\chi \beta^{2} \mu^{2} \frac{\partial^{2}}{\partial t^{2}}\left[\frac{\partial^{2} U}{\partial y^{2}}-\mu^{2} \frac{\partial^{4} U}{\partial y^{4}}\right]+\chi V^{2} \frac{\partial^{2} W}{\partial y^{2}} \\
+\chi \beta V \sqrt{m}\left(2 \frac{\partial^{2} W}{\partial t \partial y}-\frac{\partial U}{\partial t} \frac{\partial^{2} W}{\partial y^{2}}-\frac{\partial W}{\partial y} \frac{\partial W}{\partial t} \frac{\partial^{2} W}{\partial y^{2}}-\frac{\partial W}{\partial y} \frac{\partial^{2} U}{\partial y \partial t}\right) \\
-\mu^{2} \chi \beta V \sqrt{m}\left[2 \frac{\partial^{4} W}{\partial t \partial y^{3}}-\frac{\partial^{3} U}{\partial t \partial y^{2}} \frac{\partial^{2} W}{\partial y^{2}}-\frac{\partial U}{\partial t} \frac{\partial^{4} W}{\partial y^{4}}-\frac{\partial^{3} W}{\partial y^{3}} \frac{\partial W}{\partial t} \frac{\partial^{3} W}{\partial y^{2} \partial t} \frac{\partial^{2} W}{\partial y^{2}}-\frac{\partial W}{\partial y} \frac{\partial W}{\partial t} \frac{\partial^{2} W}{\partial y^{2}}-\frac{\partial^{2} W}{\partial y^{3}} \frac{\partial^{2} U}{\partial y \partial t}-\frac{\partial W}{\partial y} \frac{\partial^{4} U}{\partial y^{3} \partial t}\right]=0
\end{array}\right.
$$

\section{Solution to the instability of SWCNT conveying fluids}

The deflection of the tube can be approximated by $\mathrm{N}$ modes using the Galerkin's procedure.

$W=\sum_{n=1}^{N} w_{n}(t) \varphi_{n}(y), \Psi=\sum_{n=1}^{N} \psi_{n}(t) \phi_{n}(y)$,

In this study, let us restrict ourselves to the case $\mathrm{N}=2$. This is due to the fact that the response of the nanotube at the first and second modes is dominant. In order to satisfy the boundary conditions, the following basis functions $\varphi_{\mathrm{n}}(\mathrm{y})$ and $\phi_{\mathrm{n}}(\mathrm{y})$ satisfying the boundary conditions are used:

$\varphi_{n}(y)=\sin (n \pi y) ; \quad \phi_{n}(y)=\cos (n \pi y)$;

A non linear differential system is then obtained on $w_{n}$ and $\psi_{n}$ and which can be solved by a numerical procedure

\section{Numerical results and discussions}

Numerical results are presented using the following geometrical and material properties: $a=1.42 A^{\circ}, h=0.066 \mathrm{~nm}, E=5.5 T P a, v=0.19$,

$d=0.678 \mathrm{~nm}, \rho_{f}=1 \mathrm{~g} / \mathrm{cm}^{3}, m_{f}=2.94 \times 10^{-16} \mathrm{~kg} / \mathrm{m}$,

$\rho_{s}=2.3 \mathrm{~g} / \mathrm{cm}^{3}, m_{c}=3.23 \times 10^{-16} \mathrm{~kg} / \mathrm{m}, J_{f}=1.69 \times 10^{-35} \mathrm{kgm}$,

$J_{c}=3.72 \times 10^{-35} \mathrm{kgm}, V_{n}=V_{0} \sqrt{\rho_{s} / E}$,

The nonlinear free vibration of simply supported singlewalled carbon nanotubes conveying fluid based on nonlocal elasticity theory can be studied. For linear analysis, the evolutions of the real and imaginary components of dimensionless linear frequency parameter with the dimensionless flow velocity are presented in "figure 1" for the first two modes of an S-S CNT.

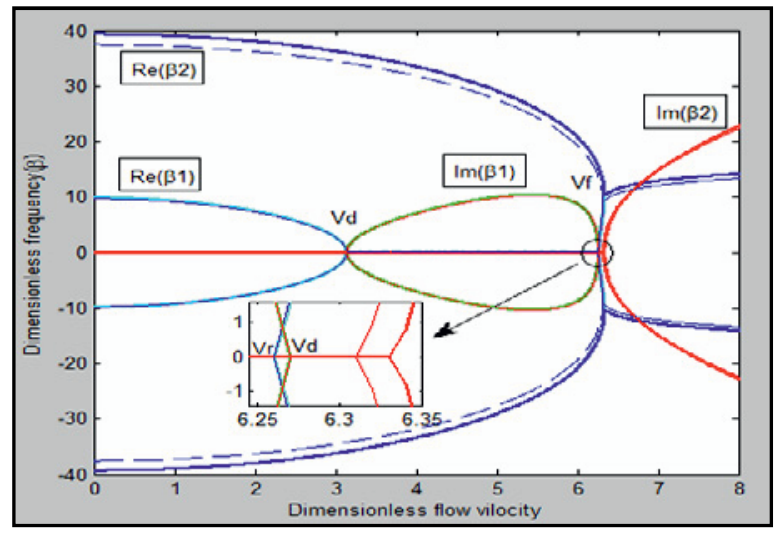

Fig1. Variations of the real and imaginary parts frequency for modes I and II of simply supported CNT with the flow velocity with and without nonlocal parameter.

Fig1. shows the flutter and divergence velocities for the considered S-S CNT. The real frequency parts decrease with increasing of the nonlocal parameter $\mathrm{e}_{0} \mathrm{a} / \mathrm{L}$. The divergence in the first mode occurs at critical velocity $\mathrm{V}_{\mathrm{c}} \mathrm{d}=3.3$ and in the second mode at $\mathrm{V}_{\mathrm{c}} \mathrm{d}^{\mathrm{d}}=6.3$. The flutter instabilities occur at $\mathrm{V}_{\mathrm{c}}^{\mathrm{F}}=6.4$ and the coalescence between the first-second modes are obtained

\section{Acknowledgements}

The authors would like to acknowledge the support provided by the "convention CNRST-CNRS" (Morocco-French), SPM06/12 and the technical and financial supports of the King Abdulaziz University, under grant No. (7-4 - 1432/HiCi).

\section{Conclusions}

The nonlinear free vibration modeling of simply supported single-walled carbon nanotubes conveying fluid based on nonlocal elasticity theory is formulated. The Timoshenko beam model and the Hamilton principle are utilized in the modeling of the system with considering the von Kármán geometric nonlinearity. The frequencies and amplitudes of the nonlinear vibration of the SWCNT are obtained by Galerkin's method. The influences of nonlocal parameters and flow velocity on the resonant frequency are discussed.

\section{REFERENCES}

[1] A.C. Eringen and DGB Edelen, Int. J. Eng. Science10, pp. 233-248.

[2] J. Yang, L. L. Ke, S. Kitipornchai, Physica E 42, 2010, pp 1727-1735.

[3] Bo Wang, Zi-chen Deng, Kai Zhang, J. Appl. Math. Mech. -Engl. Ed., 34(3), 2013, pp 269-280

[4] Haw-Long Lee, Win-Jin Chang, Physica E: Volume 41, Issue 4, 2009, pp 529-532.

[5] A. Azrar, L. Azrar and A. A. Aljinaidi, Revue de Mécanique Appliqué et Théorique, Vol. 2, 5 pp 475-484, 2011.

[6] A. Azrar, L. Azrar, A. A. Aljinadi and M. Hamadiche, Advanced Materials Research Vol. 682 pp 153-160, 2013 